\title{
Vortragsabend
}

der Akademie der Wissenschaften zu Götringen

im Niedersächsischen Landtag in Hannover

\section{Am Ende das Buch - Lesen im digitalen Zeitalter}

\author{
GERHARD LALER
}

9. Oktober 2012

Wir Menschen können über kaum envas anderes so schr erstaunen wie über uns selbst. Zum Beispiel darüber, womit wir unsere Zeir verbringen. Wenn der Druck der alltäglichen Geschärigheir machlasst und wir uns aussuchen können, was wir als Nichstes tun und welchen Leidenschaften den großen und kleinen - wir denn nachgehen wollen, dann ennwickeln wir Neigungen, die auch die Wissenschaften erstaunen. Und so kam es, dass eine Gruppe von Sozialpsychologen genauer wissen wollte, was wir dann tun, wenn wir aus uns selbst heraus agieren dürfen. Um das genauer herauszufinden, verteilten die Wissenschaftler' Blackberry-Telefone an die mehr als 200 Teilnehmer ihrer Studie. Über 14 Stunden des Tages verteilt und über mehr als eine Woche hinweg wurden die Teilnehmer mehrfach am Tag befragt, welche Leidenschaften sie in den letzten Stunden erlebt oder gepflegt hatten. Die Rückmeldungen wurden von den Wissenschaftlern in Typen der Leidenschaften und Wünsche klassifiziert, Nachfragen wurden gestellt, zum Beispiel danach, ob die Leidenschaften mit anderen äußeren oder auch persönlichen Zielen konfligierten und vor allem ob sie den Leidenschaften widerstehen konnten oder nicht. So kamen mehrere tausend Mikro-Episoden der Leidenschaften zusammen und natürlich waren die häufigsten solche, die etwas mit Essen und Trinken, Schlafen und Sex zu run hatten. Das wird niemanden erstaunen. Erstaunlicher fanden die Wissenschaftler hingegen, was als nächste Leidenschaften folgte, und das waren Leidenschaften um alles das, was mit Medien im weitesten Sinne zu tun hat. Nichts fiel den Teilnehmern der Studie so schwer, wie der Versuchung zu widerstehen, den Fernseher anzumachen, ein Buch oder eine Zeitschrift zu lesen, das Internet zu nutzen oder Computer zu spielen. Die Wissenschaftler folgern aus ihren Befunden, dass vermutlich wir Menschen generell eine schier nicht zu unterdrückende Neigung dazu ha-

1 Wilhelm Hofmann, Kathleen D. Vohs, \& Roy F. Baumeister, What people desire, feel conflicted about, and try to resist in everyday life, in: Psychological Science 23, 6 (2012), S. 582-588. 
ben, uns in Geschichten zu verlieren, seien es Herzblatt-Geschichten oder Abenteuererzählungen, Talk- und Realityshows oder mehrteilige Literaturverfilmungen, das Surfen im Internet oder das Durchblättern von KlatschZeitschriften, Computerspiele oder Sportereignisse. Immer geben wir dem Wunsch nach, noch einer weiteren Geschichte zu folgen, obwohl weder Hansi Hinterseer noch Winnetou, Werther oder Harry Potter, Jane Eyre noch Holly Golightly oder Anna Karenina, Jürgen Klopp oder Lady Gaga unmittelbar zu unserem Leben gehören und es eigentlich uns gleich sein könnte, ob es die nun gibt oder auch nicht. Aber wir sind anders und eben das ist erstaunlich. Es macht uns erheblich was aus, was alle diese Figuren und Personen so machen, solange sie in Geschichten vorkommen. Und diesen Geschichten, sie mögen noch so trivial sein, können wir kaum widerstehen, auch wenn wir wissen, dass viele davon nur erfunden sind oder es eigentlich gar nicht unser Leben beeinflussen sollre, ob nun dieser Hans seine Grete kriegt oder jener Fußballer diese Torchance doch genurzr haben müsste. Nichts hängt daran und doch alles.

\section{Zur Kritik der Medien}

Diese kleine anthropologische Vorbemerkung illustriert eine Eigenschaft von uns Menschen, von der es höchst unwahrscheinlich ist, dass sie demnächst verschwindet. Und wenn diese These vom Hunger nach Geschichten in ihrer Schlichtheit einige Plausibilität für sich hat, dann vielleicht auch die These, dass sich dieser Hunger auch immer neue Medien suchen wird, die ihn zu stillen versprechen. Nicht weniger, sondern mehr Geschichten und mehr Medien umgeben uns heute, gleich ob digital oder analog. Und hier setzt nun der kulturkritische Gegen-Blick an. Neue Medien ziehen Kritik auf sich, die eine Gegenrechnung aufmachen und behaupten, dieser Hunger nach neuen Medien würde unsere menschlichen Grundzüge entstellen. Der Computer ist ein solches neues Medium, das den Hunger nach allerlei Geschichten stillt und der Kritik entsprechend ausgesetzt ist, vor allem der, das Buch zu erdrücken. Seit Mitte der 90er Jahre, seit dem der Computer immer rascher die Gesellschaft in immer weiteren Lebensbereichen durchdringt, desto sicherer ist sich die Kritik, dass der Computer eben dieses Humanum abtöte. Das neue Medium entfalte eine fatale Wirkung besonders auf die Jugend und oft auch auf die Frauen. Die Beobachter solcher Veränderungen, zumeist Männer in angesehenen Bildungsinstitutionen, verweisen auf eigene Erfahrungen und auf Expertenstudien, die alle zeigen, dass wir ständig abgelenkt, süchtig nach 
dem neuen Medium werden und darüber wesentliche Anforderungen des Lebens versäumen. Nervenzellen sterben ab und wir verlieren ganz wörtlich den Verstand. Dick und dumm verfehlen wir, was wir als Menschen sein könnten. Hören wir einer solchen Medienkritik genauer zu:

„Wir wollen einmal die neue Medienwelt an einem sonnräglichen Vormittag belauschen. - Sehen Sie dort den Jungen auf dem Sofa: Wie andächrig! Den Kopf auf die Hand gestützt. Vor ihm ein Buch. Die Augen verschlingen die Schrift. ,Vielleicht ein Schulbuch oder ein gutes Buch aus dem väterlichen Bühèrregal::" - Ein iPad. Er spielt eine Stunde, er spielt noch eine. Endlich erhebe er sich, will sich anziehen und raus zu seinen Freunden gehen. Doch die Szene is gaar zu interessant. Er serzt sich wieder und spielt weiter. - Die Sonne schein drauken. Er siehr begierig der Aufösung des Knotens entgegen und spielt weiter - Die Sonne ist untergegangen."

Sie haben es bemerkt. Das ist gar keine aktualle Kritik am Verhalten unserer Tage, sondern eine Medienkritik, die 200 Jahre alt ist und dem Buch galt. Im Original heifst es mit Blick auf den damals gerade populären Erfolgsroman der Goethezeir, den Räuberroman Rinaldo Rinaldini:

Wir wollen einmai die şalante Welt an einem sonntäglichen Vormittag belauschen. -
Sehen Sie dort eine Dame auf dem Kanapee: Wie andächtig! Das Köpfchen auf die
Hand gestürzt. Vor ihr ein Buch. Die Augen verschlingen die Schrift. ,Vielleicht ein
Morgengebet oder Materialien zur häuslichen Erbauung?!" - Rinaldo Rinaldini. Sie
lieser eine Scunde, sie lieset noch eine. Endlich erhebt sie sich, will ihre Toilette machen
und in die Kirche gehen. Doch die Scene ist gar zu interessant. Sie setzt sich wieder und
liest weiter. - Die Glocke ruft. Sie sieht begierig der Auflösung des Knotens entgegen
und lieset fort. - Die Kirche ist aus. "

Heute wären viele froh, wir hätten nur über das exzessive Lesen von Räuberromanen Grund zur Klage. Stattdessen sei die Situation viel schlimmer, so etwa der deutsche Philosophische Fakultätentag, ${ }^{3}$ weil im Zeitalter des Computers die Fähigkeit selbst unter Studenten verloren gegangen sei, sich länger auf einen komplexeren Gegenstand zu konzentrieren und ihn formulieren zu können. Verfall der Lesekompetenz, ja weltweites Lesenverlernen konstatieren Kritiker wie Nicholas Carr in seinem vielbeachteten Artikel von $2008 .{ }^{4}$ Wir verlieren, so Carr, die Fähigkeit zum vertieften Lesen, dem „Deep Reading“, wie er sie nennt, und das betreffe nicht nur die Literatur, sondern auch die Auseinandersetzung mit anderen Künsten und

2 Johann Wilhelm Barcholomäus Rußwurm, Prognostikon über das Kirchengehen, in: Mecklenburgisches Journal (August 1805), S. 81-127, S. 91, zitiert nach Holger Dainat, Abaellino, Rinaldini und Konsorten. Zur Geschichte der Räuberromane in Deutschland, Tübingen 1996, S. 93.

3. http://www.heise.de/tp/blogs/6/152450.

4 Monarszeitschrift The Atlantic (Juli/August 2008), høp://www.theaclantic.com/magazine/ archive/2008/07/is-google-making-us-stupid/306868/. 
den Wissenschaften. Und gewiss nachdenkliche Schriftsteller wie Jonathan Franzen beklagen denn auch einen Verfall des Lesens angesichts von iPad, Amazon Kindle und Internet. ${ }^{5}$ Stirbt also das Buch und mit ihm die Fähigkeit zu lesen?

Die Antwort auf diese Frage kann nicht so einfach sein, wie es die kulturkritischen Klagen nahelegen. ${ }^{6}$ Denn deren notorische Unterkomplexität ist schon Teil des Problems, um das es im Folgenden gehen wird. Macht man es sich nämlich erheblich zu einfach mit seiner Kulturdiagnose, dann tendieren auch Zurückweisungen dieser Thesen zu nur umgekehrten Trivialitäten. Denn natürlich ist es einfach, zu zeigen, wie viel Schlichtheit in solchen Verfallsklagen steckt und wie wenig diese über die Addition bloßer Meinungen hinauskommen. Ich möchte Ihnen stattdessen vorschlagen, genauer hinzusehen, was es mit Buch und Lesen im digitalen Zeitalter auf sich hat. Der erste Schritt ist ein historischer. Schauen wir uns genauer an, was Medienkritik meint, wenn sie vom Verfall des Lesens redet. Dann sehen wir uns näher die Leserinnen und Leser unserer Gegenwart an, wie sie mir E-Book und Compurer ungehen und versuchen schließlich drittens rorläufig abzuschärzen, ob das Buch noch eine Zukunft hat und was das fur das Leson heifir.

\section{Geschichte der Medienkritik}

Medienkritik ist alt und meist verweist man mindestens auf Plato, um zu betonen, dass Klagen über den Verfall der Jugend und Kritik der medialen Erneuerung eine lange Tradition haben. Das ist richtig, verstellt aber den Blick auf die Besonderheiten der Moderne und des Buchs. Um das zu verstehen, schlage ich eine idealtypisierende Unterscheidung in drei Stadien der Medienkritik vor. In dieser Idealtypik steht die Antike für die Kritik an der Einführung des neuen Mediums der Schrift. Plato erzählt in seinem Dialog Phaidros den Theuth-Mythos, die Geschichte von der Einführung der Schrift im Alten Ägypten. Statt die Erfindung der Schrift zu feiern, beklagt Sokrates den mit der Schrift unvermeidlich einsetzenden Verfall:

„Denn diese Erfindung“, lässt Plato Sokrates sagen, „wird der Lernenden Seelen vielmehr Vergessenheit einflößen aus Vernachlässigung des Gedächtnisses, weil sie im Vertrauen auf die Schrift sich nur von außen vermittels fremder Zeichen, nicht aber

Anita Singh: Jonathan Franzen - E-Books are damaging society, in: The Telegraph (29.1.2012), hrcp://www.telegraph.co.uk/culture/hay-festival/9047981/Jonarhan-Franzen-e-books-aredamaging-society.html.

Vgl. zur Übersichr Jim Macnamara, The 21st Century Media (R)evolution: Emergent Communication Practices, New York 2010. 
innerlich sich selbst und unmittelbar erinnern werden. Nicht also für das Gedächtnis, sondern nur für die Erinnerung hast du ein Mittel erfunden, und von der Weisheit bringsr du deinen Lehrlingen nur den Schein bei, nichr die Sache selbst. Denn indem sie nun vieles gehört haben ohne Unterricht, werden sie sich auch vielwissend zu sein dünken, da sie doch unwissend größtenreils sind, und schwer zu behandeln, nachdem sie dünkelweise geworden statt weise."

Dieses antike Argument, nur den Schein, nicht aber die Sache selbst zu verstehen, läuft durch die Jahrhunderte bis heute, wenn wir darüber klagen, dass Google unser Gedächtnis ersetze und Schüler zwar alles nachschlagen, aber nichts verstanden haben. Auch wenn kaum noch jemand gegen die Einführung der Schrift polemisieren und der antiken Höherschätzung der Geheimlehren für die ganz, ganz wenigen das Wort reden würde, so findet sich das Argument auch heute. Das neue Medium Computer lenke uns von der wesentlichen Durchdringung der Gegenstände ab. Die Schüler seien zwar vielwissend, hätten aber deshalb noch lange kein Wissen, - das isc die moderne Variante der antiken Hochschätzung esoterischer Arkana gegenüber den bloßen Meinungen der Vielen. So gängig ein solches, gewiss altes Argument ist, so wenig ist diese antike Medienkritik schon mit der unseren heure gleichzusetzen.

In einer zweiten idealtypischen Konzeprualisierung lässt sich die mitrelalterliche und besonders frühneuzeitliche Kritik der Bücher von dieser antiken Kricik der Schrift uneerscheiden. Hier ise niche die Schrift strittig, denn das würde dem christlichen Offenbarungsanspruch widerstreiten, sondern die Frage, wer die Bücher lesen darf. Solange nur sehr wenige schreiben konnten, die Materialien für die Codices teuer und selten waren, beschränkten sich etwa die mittelalterlichen Universitäten darauf, die Studenten beim Verlassen der Universitätsstädre zu kontrollieren, damit sie keine nur an diesem Ort vorhandene Bücher mitnahmen und damit Konkurrenzstandorte stärker konnten. Mit der Reformation und dem Buchdruck wird freilich etwa ab dem 16. Jahrhundert das Lesen höher bewertet und leichter zugänglich. Weil Lesen getrennt vom Schreiben unterrichtet wurde, übersprangen das Lesen, manchmal auch das Schreiben und das einfache Rechnen die Mauern der Lateinschulen und Universitäten. Nun beginnen auch die Schlachtersknechte und Karrenführer, die Handwerkersfrauen und ihre Töchter mal in Winkelschulen, mal in landesherrlichen Anstalten wenigstens etwas Lesen zu lernen, mit freilich großen regionalen Unterschieden. Wenn in Florenz fast jedes zweite Kind lesen lernte und in einer Stadt wie Lübeck im 16. Jahrhundert mehr als 60 win- 
zige und manchmal auch größere Schulen gezählt werden konnten, so gab es viele Regionen Europas, wo selbst der Pfarrer nicht recht lesen konnte. "Lesen können hatte sich noch nie also so nürzlich erwisen wie jetzt", schreibt der Grammatiker Valtentin Ickelsamer in seiner Schrift Die rechte weis auffs kürtzist lesen zu lernen 1527 , „da jeder deshalb lesen lerne, damit er GOTTES Wort und Auslegung selber lesen und umso besser darüber urtheilen könne". ${ }^{8}$

Aber das ist zumal im Zeitalter der Konfessionalisierung so eine Sache, wenn jeder selber liest und selbst urteilt, was Gotres Botschaft sei. Und so serzt die frühneuzeitliche Medienkririk daran an, dieses Selbstlesen zu regulieren, und zwar durch die Instanz des Hausvaters. Er, der über die formal höchste Bildung verfügt, entscheider darüber, wer wie viel lesen lernen darf und wer welche Bücher in die Hand bekommt. Noch für Christian Wolff, den großen Aufklärer und Schüler Leibniz', war es selbstverständlich, dass der Hausvater die Lesewut und die daraus folgende Gefahr, auf falsche Gedanken zu kommen, zu regulieren habe und also entscheide, was seine Ehefrau, die Kinder und das Gesinde jeweils lesen dürfen." Auch hier kehrt das Argument wieder, dass die unkontrollierte Lektüre moralisch verheerend wirke, weil insbesondere Frauen und die Jugend ohne männliche Anleitung nicht verstünden, was sie da lesen. Noch der Philosoph Fichte, der sonst die Revolution auch in Bildungsdingen gefordert hat, sieht die Gefahr eines Lesens „ohne Anhalt“, wie er es nennt. Von diesen Leserinnen und Lesern sagt Fichte: „Nirgends können sie in diesem rastlosen Fluge anhalten, um mit sich selber zu überlegen, was sie denn eigentlich lesen, "10 so dass es derjenigen bedarf, die vor diesen Gefahren immun sind, eben die Hausväter. Die verstehen alles und leiten also an. Auch diese frühneuzeitliche Medienkritik reicht weit in unsere Tage hinein, etwa in die Warnung vor der sirtlich verheerenden Wirkung des Farbfernsehens auf die Frau in den 70er Jahren des 20. Jahrhunderts.

Aber noch etwas muss dazukommen, damit unsere spezifisch moderne Lesekritik formuliert werden kann, die über die Kritik am mangelnden Verstehen und den moralisch problematischen Folgen des unkontrollierten

Valentin Ickelsamer, Die rechte weis auffs kürtzist lesen zu lernen, Erfurt 1527, ziriert nach Gerhard Lauer, Die Bildung des Menschen. Zur Ideengeschichte eines unwahrscheinlichen Begriffs. Lemmermöhle, D., \& Hasselhorn, M. (Hg.). Bildung - Lernen. Humanistische Ideal, gesellschaftliche Notwendigkeiten, wissenschaftliche Erkenntnisse. Göttingen 2007, S. 59-78, S. 64. Chrisrian Wolff, Vernünftige Gedancken von dem gesellschaftlichen Leben der Menschen insonderheit dem gemeinen Wesen, 4. Auf. Frankfurt, Leipzig 1736 (Neudruck 1975), \$\$192ff.

10 Johanı Gotclieb Fichre, Die Grundzüge des gegenwärtigen Zeitalters [1805], in: ders., Fichtes Werke: Zur Politik, Moral und Philosophie der Geschichte, Hg. von I. H. Fichre, Bd. 7, Berlin 1845 (Neudruck 1965), S. 89. 
Lesens hinausgeht. Das ist die Bedeutung des Buchs als Freund. ${ }^{11}$ Erst im 18. Jahrhundert und dann in der Masse im 19. Jahrhundert, als der Buchdruck durch Rotationsdruckmaschinen und billiges Papier erschwinglich für Jedefrau und Jedermann wird, ist das Buch mehr als nur ein Instrument der Erbauung, der gelegentlichen Ablenkung oder ein Wissensspeicher. Es wird zum Freund, demjenigen, der ausspricht, was man vage denkt und noch undeutlicher fühlt, das einen versteht und dieses ,je ne sais quoi“, dieses „ich weiß nicht, was soll es bedeuten“ auszudrücken vermag, was sonst niemand um mich herum kann. Und wenn die ganze Welt mich nicht versteht, mein Buch tut es. Mit ihm rede ich, über es schreibe ich meine Briefe und vergieße meine Tränen darüber.

Es kam einem Erdbeben gleich, als der Erfolgsautor des 18. Jahrhunderts, eben Rousseau, seinen Briefroman Julie, ou la Nouvelle Héloïse 1761 veröffentlicht hatte. Nicht dass es Liebesgeschichten etwa als Ritterromanzen nicht früher schon gegeben hätte, über deren Lektüre bekanntlich Don Quichote seinen Verstand verloren hat. Aber mit Rousseau beginnt weithin sichtbar jenes Lesen, das Jürgen Habermas die meditative Privatlektüre genannt hat. ${ }^{12}$ Enthusiastische Briefe an den Freund Jean-Jacques, wie die Leser Rousseau damals genannt haben, bezeugen, dass das Lesen dieses Romans Rousseaus Lesern alles war und ihr Herz geöffnet hat. Ihm schüttet beispielsweise der Leser und Verleger Panckoucke sein ganzes Herz in einem Brief aus:

"Ihre göttlichen Werke, Monsieur, sind ein alles verzehrendes Feuer. Sie haben meine Seele durchdrungen, mein Herz befestigt, meinen Verstand erleuchrec. Lange Zeir ging meine Vernunft, die den täuschenden Illusionen einer stürmischen Jugend anheimgefallen war, auf der Suche nach der Wahrheir in die Irre. Ich strebte nach Glück und es entzog sich mir. [...] Das Studium einjger modernen Auroren harte mich in meinen Uberlegungen bestärkt, und in meinem Herzen war ich schon durch und durch ein Schufr. ohne aber noch exwas geran zu habern. über das ich hätte erröten müssen. Ich brauchte einen Gott, éinen mächrigen Gorr. der mich von jenem Abgrund fortzog. und Sie. Monsieur, sind der Gort. der dieses Wunder vollbracht hat. Die Lekrüre ihrer Hélożse hat vollender. was Ihre anderen Werke schon begonnen hatren. Wie viele Tränen habe ich darüber vergossen! Wie viele Seufzer getan und Qualen erlitten! Wie oft sah ich meine eigene Schuld. Seit ich Ihr gesegnetes Buch gelesen habe, bin ich in Liebe zur Tugend encbrannt, und mein Herz, das ich schon erloschen

11 Katja Mellmann, Emotionalisierung - Von der Nebenstundenpoesie zum Buch als Fieund. Eine emotionspsychologische Analyse der Literatur der Aufklärungsepoche, Paderborn 2006.

12 Jürgen Habermas, Strukiurwandel der Öffentlichkeit. Untersuchungen zu einer Kategorie der biirgerlichen Gesellschaft, Darmstadt ${ }^{5}$ 1971, S. 60-69. 
glaubte, schlägt wackerer denn je. Das Gefühl hat mich wieder: Liebe, Mitleid, Tugend, holde Freundschaft haben auf immer meine Seele erobert. ${ }^{\text {“13 }}$

Leser werden zu Fans, das ist das Moderne an diesem neuen Lesen. Man pilgert an Rousseaus Zufluchtsort in Montmorency, ja und man hofft nicht weniger als dort die doch eigentlich von Rousseau nur ausgedachten Figuren der Julie oder des Saint Preux treffen zu können. Hier sind wir beim Kern der spezifisch modernen Mediennutzung angekommen, bei der meditativen Privatlektüre. Erst in der Moderne lesen wir ganz selbstversunken, wir lesen allein und noch schlimmer - aus Sicht älterer Jahrhunderte - wie lesen auch leise. Lesen war bis dahin fast ausschließlich ein öffentlicher Akt, in der Moderne wird es zu einem privaten, in sich gekehrten und stillen. Das stille und das identifikatorische Lesen, das ganz in den Schuhen seiner Helden wie Rousseaus Julie, Goethes Werther oder Puschkins Tatjana geht, diese moderne Form des Lesens wird zur eigentlichen Lektüre erhoben.

Das erkennt man noch an einem anderen Umstand. Erst in der Moderne nämlich wird auch das Musikhören unter medienkritischen Verdacht gestellt. War Musik bis dahin vor allem im Zusammenhang mit Spielsucht und Tanzleidenschaften verdächtig, gerät erst im 19. Jahrhundert mit der aufkommenden romantischen Musiksprache Mendelssohns und Schumanns auch die Musik in den Verdacht, dass sich die Hörer, noch bedenklicher die Hörerinnen in der Musik verlieren könnten, süchtig danach werden und nur noch ihre Stars wie etwa den Virtuosen und ersten Popstar der klassischen Musik Franz Liszt im Kopf haben könnten, sonst aber alles vergessen. Die Kritik vermutet gar, dass die Leser und Hörer nicht mehr wissen, ob sie es mit der Wirklichkeit oder mit Fiktion zu tun haben. Moderne Medienkritik problematisiert also nicht mehr nur die Frage, wie viel das Volk überhaupt lesen darf und verstehen kann. Es problematisiert den Akt des Lesens als meditative Privatlektüre als solchen und sieht in ihm die Gefahr, dass die Leserinnen und Leser vom wahren Leben abgezogen werden.

Vor dem Hintergrund dieser kurzen Geschichte des Lesens und der Medienkritik wird deutlicher, worum es in der Debatte um die Zukunft von Buch und Lesen überhaupt geht. Da sind die traditionellen Argumente, die das mangelnde Verstehen des Gelesenen beklagen und auf eine Regulierung durch zumeist männliche Bildungsautoritäten setzen. Und da sind die spezifisch modernen Argumente. Nach ihnen können die Leserinnen und Leser nicht mehr unterscheiden, ob sie in der erfundenen Welt oder

Zirierr nach Robert Darnton, Das große Katzenmassaker. Streifzüge durch die französische Kultur vor der Revolution. München 1989, S. 280. 
der wirklichen leben, ja sie verlieren sich so sehr in der erfundenen Welt, dass sie darüber alles andere vernachlässigen, die Freunde genauso wie die körperliche Bewegung und also unweigerlich dumm und dick werden. Das Argument haben also Medienkritiker wie aktuell Manfred Spitzer nicht erfunden. ${ }^{14}$ Vielmehr wird es seit dem einflussreichen Buch des amerikanischen Psychiaters Frederic Wertham mir dem sprechenden Titel Seduction of the Innocent [Verfuibrung des Unschuldigen] auch wissenschaftlich behauptet, damals - 1954 - mit Blick auf die Gewaltdarstellung in Comics. Wertham selbst wird dann in den 60er Jahren statt der Comics das Fernsehen als Hauptverantwortlichen für die moralische Verwahrlosung der Jugend ausmachen. Die Argumente bleiben aber die gleichen und gelten heute dem Computer.

Daran ist zunächst richrig, dass das identifikatorische Lesen in der Moderne zugenommen har. Genauer noch, nicht nur die Zahl der Bücher steigt seit dem 19. Jahrhundert, sondern auch die Gattungen und Genres und seit dem 20. Jahrhundert auch die Medien werden zahlreicher, die ein solches, selbstversunkenes Lesen ermöglichen. Der vermutlich angeborene Hunger nach Geschichten sucht sich immer neue Bücher in allen möglichen Formaten und Medien. Mit dem Schundgesetz von 1926 und öffentlichen Buchverbrennungen auf Schulhöfen - in der DDR noch bis in die 50er Jahre - wurde etwa gegen die neuen Medien der Heftromane angegangen, die Ende des 19. Jahrhunderts aufgekommen waren. Lesen in der Moderne ist frei, sich immer neue Formate zu suchen, um sich in ihnen zu verlieren. Kritik hat es dabei immer auf sich gezogen. Wir wissen allerdings längst, dass etwa diese Detektiv-Heftromane um Tom Shark und Harry Piel nicht nur von Kindern unter der Schulbank gelesen wurden, sondern auch von deren Vätern, was noch mehr für die amerikanischen Comics gilt. Freilich las der Papa dann nur daheim sein Abenteuerheftchen und pilgerte nur mit den engsten Freunden zu der angeblichen Villa seines Heftroman-Helden, von der er zugleich wusste, dass sie nicht existiert. Öffentlich konnte er das nicht tun. Anders gesagt: Seitdem in dieser Gesellschaft fast jeder Zugang zum Lesen und zu Büchern hat, wird nicht nur Homer gelesen, sondern auch die historischen Schmöker eines Felix Dahn, nicht nur Goethe, sondern auch die Räuberromane von Vulpius, nicht nur Max Frisch, sondern auch Comics. Der Hunger nach Geschichten kann in der Moderne vielfältiger gestillt werden und das tritt immer mehr hervor.

14 Manfred Spiczer, Digitale Demenz. Wie wir uns und unsere Kinder um den Verstand bringen, München 2012. Das Buch liegt natïrlich auch digital als E-Book vor. 
Damit sind wir bei dem ersten systematischen Punkt. Über welche Grundgesamtheit reden wir eigentlich, wenn wir über das Lesen und dessen Verfall reden. ,Grundgesamtheit 'ist ein Ausdruck der Statistik und meint die Gesamtmenge, auf die bezogen eine tatsächliche Gruppe näher untersucht wird. Das Problem ist ganz einfach: Wir wissen nicht hinreichend genau, wie viel und was vor 100 oder vor 50 Jahren gelesen wurde. Es fehlen nicht nur belastbare Zahlen, es fehlen auch klare Vergleichskategorien, um von Leseverfall reden zu können. Als es noch keinen Computer und keinen Fernseher gab, haben andere Medien wie etwa Heftromane jene Genres abgebildet, die seit den 60er Jahren, wie Studien in den Niederlanden gezeigt haben, in anderen Medien wie dem Fernseher oder heute dem Computer auftauchen. ${ }^{15}$ Insofern wurde vermutlich früher mehr gelesen, wenn damit gedruckte Sachen gemeint waren, deren Geschichten heute eher gehört oder vor allem gesehen werden. Der Hunger nach Geschichten hatte damals noch nicht so viele verschiedene technische Medien zur Verfügung, über die wir heute verfügen. Die gedruckten Geschichten sind aus den Familienblättern des 19. Jahrhunderts in die Heftromane und heute in die Computerspiele gewandert. Verändert hat sich sicherlich die Buchkultur, aber deshalb noch nicht unbedingt die Lesekultur. Eine seriöse Forschung müsste also erst einmal sagen, was sie vergleicht, wenn sie vom Verfall des Lesens spricht. Vermutlich ist die Grundgesamtheit der Geschichten, in die wir eintauchen, nicht so viel anders als früher, nur die Formate sind andere.

Und ein zweites Argument der spezifisch modernen Medienkritik bleibt eigentümlich ungenau. Das ist die These von der mangelnden Unterscheidung von Wirklichkeit und Fiktion. Schicken nicht Zuschauer körbeweise Babywäsche an Serienstars, wenn diese in ihrer Rolle schwanger werden, aber nicht in der Wirklichkeit? Schreiben nicht Zuschauer an Prof. Brinkmann über ihre Krankheiten und hoffen, dass er ihnen so schön helfen kann wie in der Fernsehserie Schwarzwaldklinik? Die Antwort ist: Ja, das tun Fans seit den Tagen Jean-Jacques Rousseaus, aber - und das ist das „nein“-, nein, sie wissen dabei zugleich, dass Prof. Brinkmann nur ein Schauspieler ist und Rousseaus Figur Saint Preux nie gelebt habt. Selbst Kinder haben im Vorschulalter schon ein klares Verständnis, was eine er-

Z.B. Frank Huysmans, De openbare bibliotheek in Nederland en de veranderende leesculruur sinds 1975, in: Jaarboek voor Nederlandse Boekgeschiedenis (2007), S. 179-192; Adriaan van der Weel, Convergence and its discontents: From a book culture to a reading culture, in: Logos 20 (1-4) (2010), hrtp://www.let.leidenuniv.nl/wgbw/research/Weel_Arricles/AvdW_eBooks_Logos.pdf. 
fundene Welt von der wirklichen unterscheidet. ${ }^{16}$ Sie mögen noch so viele Superman-Heftchen gelesen haben und stürzen sich doch nicht von Dächern. Ja mehr noch, selbst kleine Kinder wissen, dass Robin in die erfundene Welt von Batman gehört, aber Spongebob nicht zu dieser Welt zu zählen ist. ${ }^{17}$ Sie bilden sehr sicher eigene Ordnungen der ausgedachten Welten und sind dann auch schon in der Lage, etwa die Regeln eines Ritterspiels mit denen eines Science Fiction-Genres zu kombinieren. Das Verhalten von Fans ist nicht mit deren Einschätzung der Wirklichkeit zu verwechseln, wie es die Medienkritik gerne tut, sonst wären Fans schon ausgestorben.

\section{Was ändert sich gerade}

Damit sind wir in der Gegenwart angekommen. Was ändert sich gerade unter den Bedingungen der digitalen Gesellschaft, was ist anders geworden? Verschwindet das Buch, wird nicht mehr gelesen und werden wir in der Folge also immer dümmer, dicker und asozialer, mindestens unsere Jungen? Meine Antwort auf diese Frage ist ein Nein, das ich Ihnen näher erläutern möchte, und zwar gerade deshalb, weil diese pauschale Medienkritik die wesentlichen Probleme im Umgang mit Buch und Medien gerade verstellt. Der Reihe nach.

Werden wir immer dümmer, wie es die Medienkritik suggeriert oder im Gegenteil eher intelligenter? Eben der Frage hat sich seit den 80er Jahren der neuseeländische Politologe James Flynn zugewandt und erstmals in Langfriststudien untersucht, wie sich der in Intelligenztests messbare IQWert in 14 Industrienationen entwickelt hat. Flynn, und inzwischen nicht nur er, kann nachweisen, dass der Anregungsreichtum moderner Industrienationen, die bessere Ernährung, verbesserte Schulbildung und eine Reihe weiterer Faktoren den messbaren Intelligenzquotienten ansteigen lässt. ${ }^{18}$ Insofern werden wir eher intelligenter als dümmer. Um gleich Missverständnissen vorzubeugen: Wir reden hier über messbare IQ-Werte, nicht unbedingt davon, dass wir Menschen klüger oder moralisch deshalb schon

16. Kleine Kinder unter vier Jahren dagegen verstehen nicht, was auf einem Bildschirm passiert. Daher ist Fernsehen für diese Altergruppe grob schädlich, vgl. Ari Brwon and the Council on Communications and Media, Media Use by Children Younger Than 2 Years, in: Pediatrics 128 (2011), S. 1040-1045, http://pediatrics.aappublications.org/content/128/ 5/1040.full.pdf+html.

17 Deena Skolnick \& Paul Bloom, What does Batman think about Spongebog? Children's understanding of the fantasy/fantasy distinction, in: Cognition 101 (2006), S. B9-B18, http://www.yale.edu/minddevlab/papers/skolnick\&bloom\%20cognicion.pdf.

18 James Flynn, Are we getting smarter? Rising IQ in the Twenty-First Century, Cambridge 2012. 
besser würden. Auch ist dies keine lineare Progression, so dass die Menschen in Industrienationen immer weiter in ihrer Intelligenz fortschreiten würden. Zum einen ist der Zuwachs begrenzt, weil unser Gehirn zwar plastisch, aber nicht beliebig plastisch ist. Keine Sorge also, dass demnächst Kinder mit Doppelgehirnen geboren würden. Noch gleicht die Entwicklung nur einem nach oben weisenden Pfeil. Es gibt Hinweise, dass sich diese Entwicklung seit den 90er Jahren in einigen Ländern mindestens abgeschwächt hat, in anderen steigt die Intelligenz weiter. Wenn Medienkritiker wenigstens die Abschwächung auf den Computer und das Internet zurückführen, dann ist zu sagen, dass gerade das Internet erst nach der Jahrtausendwende eine rasant wachsende Rolle in den Industrienationen spielt und daher kein Kandidar für die Erklärung der nicht immer bloß steigenden Intelligenz sein kann, einmal ganz abgesehen davon, dass ein neues Medium auch gar nicht sofort eine solche Größe wie die Intelligenz beeinflussen kann. Es gilt aber, dass die mir der Industrialisierung einhergehende Durchdringung der Gesellschaft mit immer neuen Medien und Medienformaten nicht in eine gesamtgesellschaftliche Verdummung geführt hat. Wir müssen eher mehr lesen, komplexere Abläufe verstehen und mehr Sprachen heute sprechen, gerade im digitalen Zeitalter. Und das alles macht eher ein bisschen gescheiter als dümmer.

Noch genauer: Die digitale Welt macht die ein bisschen gescheiter, die sowieso ganz gut im Lesen und nicht nur im Lesen sind. Denn ein ganz erheblicher Teil der Leserinnen und Leser hat gar kein Problem mit den neuen Medien. Weder das Aufkommen der Heftromane, des Rundfunks oder des Fernsehens und selbst der digitalen Medien überfordert alle. ${ }^{19}$ Noch nie gab es so viele Bücher, so viele Genres, so viele Medien und so viele Menschen, die damit ziemlich gut zurechtkommen und in derselben Zeit mehrere Medien nutzen als frühere Generationen. ${ }^{20}$ Wir nennen sie etwas abstrakt auch Mehr-Medienbenutzer. Sie lesen, sie hören Rundfunk, wählen ihr Fernsehprogramm aus, haben viele DVDs und sind auch unterwegs online, lesen ihre Mails auf dem Handy. Und eine überregionale Tageszeitung haben sie auch noch abonniert. Ob sie die auf dem iPad lesen oder gedruckt, ist mehr eine Frage der Gelegenheit denn der Einstellung. Manche schreiben Blogs, andere twittern, andere wieder schreiben für Wiki-

19 Eszter Hargittai, W. Russell Neumann \& Olivia Curry, Taming the Information Tide. Perceptions of Information Overload in the American Home, in: The Information Society 28, 3 (2012), S. 161-173.

20 Vgl. beispielsweise den Bericht über die Generation M (http://www.kff.org/entmedia/entmedia 030905pkg.cfm) bzw. M2 (hørp://www.kff.org/entmedia/mh012010pkg.cfm) der Kaiser Family Foundation über die Zunahme der Mediennutzung bei 8-18jährigen. 
pedia Artikel. Wer was davon tut, hat viel mit seiner oder ihrer sozialen Position in der Gesellschaft zu tun, aber überfordert sind die meisten gerade nicht. Die Zahl der genutzten Medienformate korrespondiert vielmehr in etwa mit dem Reichtum des sozialen Lebens. Viele Medien und viele soziale Kontakte, das passt zusammen, nicht umgekehrt. Die Studien der Stiftung Lesen zeigen erstaunlich stabile Muster des Lesens in der Gegenwart. Wer politisch engagiert ist, der ist das meist auch online. ${ }^{21}$ Das Internet macht also die Leute nicht politisch aktiver, vielmehr nutzen die sowieso Engagierten auch das Internet für ihre Anliegen. Für Wikipedia schreiben eher junge Männer, deren Bildungsstandard hoch ist. Diese Gruppe ist sehr klein und wächst auch nicht wesentlich, sondern sucht sich im digitalen Medium, was sie sowieso interessiert, und das können etwa Personenartikel zur Geschichte der Klassischen Philologie sein. Frauen sind eher in Genres wie den Fan-Fiction unterwegs als das Männer sind. Der Millionenerfolg des Erotikbestsellers von E. L. James Fifty Shades of Grey ist aus den weltweit Millionen von Fan-Fictions erwachsen, die eine bekannte Geschichte zur Vorlage nehmen und sie weiter- und dabei meist umerzählen. Das wird in unglaublich großer Zahl gelesen und geschrieben, aber nur ganz selten wird es auch gedruckt, noch seltener wird es ein Millionen-Bestseller. Aber geschrieben und gelesen werden solche Fan-Fictions von Tausenden, auch wenn kein Feuilleton und kein Medienkritiker diesen Leserinnen Beachtung schenkt. Der Lesemarkt besteht daher heute aus viel mehr Formaten als je zuvor. Hier steht das bibliophile Buch neben Fan-Fiction, ein schmal gewordener Kanon klassischer Werke neben schier endlosen Meeren von Geschichten, die sich an kein Format halten.

Hinter alledem sind sehr stabile Muster des Lesens auszumachen. Das fängt mit dem Vorlesen an. In Deutschland wird immer noch zu $80 \%$ den Kindern abends vorgelesen, ein gar nicht zu überschätzendes Ritual. Denn Lesen ist nun mal eine Kulturtechnik, die wie das Zähneputzen eingeübt werden muss, - daran hat sich nichts geändert. Nicht-Leser gibt es eher in der Stadt denn auf dem Land. ${ }^{22}$ Mädchen und Jungen, Frauen und Männer lesen immer noch etwas anders, die einen mehr, die anderen weniger. Emotionsbetont die einen, handlungsbetont die anderen. Mädchen

21 Claudia Rizzi, Gary S. Schaal \& Vanessa Kaufmann, Zwischen Ernst und Unterhaltung. Eine empirische Analyse der Motive politischer Aktivität junger Erwachsener im Internet, hıр://www.hsuhh.de/download-1.4.1.php?brick_id=fPRCZT2Xi9kZEPdF.

22 Petra Stanat, Hans Anand Pant, Katrin Böhme \& Dirk Richter (Hg.), Komperenzen von Schülerinnen und Schülern am Ender der vierten Jahrgangssuffe in den Fächern Deursch und Mathematik. Ergebnisse des IQB-Ländervergleichs 2011, h匹р://wרw:iqb.hu-berlin de/dara/ n/n009/LV_201]_Berichr.pdf. 
lesen auch als erwachsene Frauen noch weiter Belletristik, Jungen dagegen wechseln mit dem Erwachsenenleben überwiegend zur Lektüre von Sachtexten. ${ }^{2.3}$ Das sind sehr alte Lesemuster, vielleicht älter noch als die Neuzeit. Und eine Gruppe bleibt über die letzten Jahrzehnte in Deutschland auch stabil. Das sind die intensiven Leser, oft sind es eher Leserinnen als Leser. Sie lesen die ganze Anna Karenina, alle sieben Bände von Harry Potter und den ganzen Turm von Uwe Tellkamp, ja sie verschlingen diese Bücher. Kein Koffer kann tragen, was sie für den Urlaub als Lesestoff brauchen und so sind schon viele von ihnen zu Amazons Kindle gewechselt, der genügend Bücher in 250 Gramm digical verpacken kann. Diese begierigen Leserinnen und Leser sind nichr genau einer sozialen Schicht zuzuweisen, auch wenn sie eher weiblich denn männlich sind, eher dem Mittelstand entstammen, eher älter denn jünger sind. mal aus etablierten, mal aus zugewanderten Familien kommen, die ja auch längst einen lesenden Mittelstand ausgebildet haben, der oft und zu Unrecht übersehen wird. Unter allen diesen werden die mehr, die es nicht kümmerr, wer analog oder digital spricht. ${ }^{24}$ Das sind die unverändert stabilen Lesergruppen.

Ein erster Befund lautet also: Nicht das Lesen stirbt aus, es wird anders, diverser und das mit großer Geschwindigkeit. 1975 wurde ein Bestseller wie E. L. Doctorows Ragtime in den USA 230.000 Mal verkauft. Im Jahr 2000 verkaufte sich John Grishams Bestseller The Brethren mehr als drei Millionen Mal. Fachleute wie Gayle Feldman rechnen vor, ${ }^{25}$ dass sich im amerikanischen Buchmarkt vor 1985 nur von zwei Romanen mehr als eine Million Exemplare im Erscheinungsjahr verkaufen ließen. Im Jahr 1985 schafften es dann gleich drei Romane und noch zwei Sachbücher, mehr als eine Million Mal verkauft zu werden. Heute wundert sich niemand mehr über solche Zahlen. Viel mehr Bücher als jemals zuvor sind in immer schwindelerregenderen Auflagenhöhen im Handel. Dabei altern Romane keineswegs so schnell, wie man vermuten würde, auch wenn die Hochliteratur wie Jeffrey Eugenides Roman Middlesex trotz hymnischer Kritiken nur selten die Millionengrenze überschreitet.

Eben das ist dann auch die Schwierigkeit für die Verlage. Etablierte Geschäftsmodelle müssen immer schneller umgebaut werden. Nicht weil we-

23 Die Leseförderung hat daher die Förderung von Jungens verstärkt in den Blick genommen, vgl. die Rubrik „Leseförderung" z. B. in Eselsohr. Fachzeitschrift für Kinder-und Jugendmedien, http://www.eselsohr-leseabenteuer,de.

24 So etwa die Einschärzung von PwC: Deutscher Buchmarks: Dem E-Book gehört die Zukunft, hıр://www.pwc.de/de/technologie-medien-und-telekommunikarion/deutscherbuchmarkt-dem-e-book-gehoert-die-zukunft.jhtml.

25. Gayle Feldman, Best and Worst of Times: The Changing Business of Trade Books, 1975-2002, New York 2003, vgl. auch htcp://www.najp.org/publications/researchreports/best.pdf. 
niger Titel auf den deutschen Buchmarkt kämen, - auch ihre Zahl steigt ja von Jahr zu Jahr -, sondern weil immer schwerer zu kalkulieren ist, welcher Titel geht, welcher so durch die Decke schießt, dass er die anderen Titel dann mitträgt, die kein Erfolg werden. Denn der Markt wird kleinteiliger, in dem Maß in dem sich die Lesergruppen weiter ausdifferenzieren. Neue Genres kommen hinzu, neue Medien noch obendrauf, die meist ihre jeweiligen Leser- und Schreibergruppen haben. Dann ist schwer abzusehen, ob es besser ist, erst ein Hörbuch, eingelesen von Rufus Beck herauszubringen, das dann das gedruckte Buch auch mit sich zieht, oder umgekehrt. Und welchen Effekt es auf die Buchverkaufszahlen hat bzw. hatte, wenn Elke Heidenreich im Fernsehen ein Buch in die Höhe gehalten hat, kann jeder Buchhändler erläutern. Längst werden Stellflächen in Buchläden verkauft und im Regal gegen Geld Meter extra vermietet, um einen Titel besser herauszustellen. Das ist alles für die Verlage ein hartes Geschäft geworden, nicht weil weniger gelesen wird, sondern weil mehr, aber unterteilt in immer kleinteiligere unterschiedliche Lesergruppen mit ihren jeweiligen Ausstattungsformanten gelesen wird. Schließlich wissen circa die Hälfte der potentiellen Buchkäufer, die ein Buchgeschäft betreten, gar nicht, welches Buch sie kaufen wollen. Auf diese Leser sich einzustellen ist schwierig, und das setzt sich im Internethandel gleich noch fort. "Behavioral targeting" nennt man in der Fachsprache die Technik, mit der das Such- und Kaufverhalten von uns realen Lesern mirgeschnitren wird, um aus diesem Verhalten dann zu errechnen, dass diejenigen, die diesen Titel kaufen, auch diesen gekauft haben. Inzwischen schneiden schon die ersten E-Books mit, wie lange wir als Leser bei welchen Kapiteln verweilen, was wir überblättern und was ungelesen bleibt. Das ist die Internet-Technik, mit der sich Verlage und Buchhandel auf steigende und zugleich sich immer weiter ausdifferenzierende Lesergruppen einzustellen versuchen. ${ }^{26}$ Verlage und Buchhandel kümmert es dabei kaum noch, ob das Buch analog oder digital ist, wenn sie ihre elektronischen Spione einbauen. Buchhandel, Amazon und Google sind sich viel näher, als es der Lobbyismus-Streit um das Urheberrecht derzeit erkennen lässt.

Aber führt das nicht alles zu einer großen Trivialisierung unserer Lesekultur, die schon Plato und die Hausväter kritisiert haben? Man muss sich ja nur die Rezensionen bei Amazon oder in den sozialen Netzwerken anschauen, was da an Plattitüden geäußert wird. Ja, natürlich werden solche Gemeinplätze dort geäußert, auch über Bücher und von Lesern. „Fand ich

26 Ben Schwan, Der Spion im E-Book, hırp://www.heise.de/rr/artikel/Der-Spion-im-E-Book1674068.heml (5.9.2012). 
gut“, „hat Spaß gemacht“, „war nicht sonderlich spannend“, „ein echter Knaller" sind nicht eben Äußerungen des Hochfeuilletons. Jemand im sozialen Lesenetzwerk, Lovely Books' listet mal auf, was sie oder er liest, das klingt dann so:
„So nun mein Voting:
78sunny: Libellensommer (ich mag Kanada einfach ;))
Asu: Der Augensammler (definiriv!! ;))
Caro88: Ascheherz (da hab ich auch schon ein paar Mal davor gestanden)
Daniliesing: White Horse
ever_green: Ewiglich die Sehnsuchr (hab ich schon viel gures gehört, Plötzlich Fee hab ich mal ausgeklammere - da bin ich wohl vorbelasrer ;))
kanemabe: Eragon (ein sehr gutes Buch)
LaDragonia: Görtich verloren (rolles Buch, kann ich nur empfehlen)
licbling: The Night Circus (klingt vielversprechend)
RortenHeart: Plörzlich Fee (Die anderen kenne ich entweder nicht oder aber ich fand sie niche so gur :/)
scarlert59: Tödliche Spiele (war für mich der beste Band der Tribute von Panem :))
Si-Ne: Leider kenne ich keines der Bücher :( Aber Shades of Grey soll angeblich ja recht gut sein.
Sommerleser: darüber muss ich noch nachdenken
Sunny Rose: Zeitenzauber (klingt interessanr)
Lesewutz: Und morgen bist du tot (der Autor ist sehr gut)
Ich hoffe ich habe jetzt niemanden vergessen, sonst sagt mir bescheid :) ${ }^{\text {‘27 }}$

Es ist leicht, sich über solche Leser zu erheben. Wenn jemand ein Buch "klingt vielversprechend" findet und einen solchen Kommentar bei Amazon oder Lovely Books hostet, dann geht es nicht um Literaturkritik, sondern um die Einübung in die eigene Identität und die Suche nach Gleichgesinnten durch das Lesen. Das ist so ziemlich genau das, was auch die Leser Rousseaus getan haben, wenn sie mit Leidenschaft ihre Tränen über dem Roman vergossen und das in Briefen mitgeteilt haben. Weint um Eure Bücher, schreibt es auf und findet darüber Herzensfreunde, das ist modernes Lesen seit mehr als 200 Jahren. Und genau das passiert in den digitalen Netzwerken. So schreibt jemand über das Buch der indisch-kanadischen Autorin Shilpi Somaya Gowda:

„Als das Buch Geheime Tochter zu mir gefunden hatte, hielt ich es in den Händen und wusste noch nicht genau, was ich von dem Buch halten sollte, das Cover war schlicht,

27 http://www:lovelybooks.de/thema/Buchempfehlungen-lesen-Jeden-Monat-eineBuchempfehlung-der-anderen-lesen-Abstimmung- $\mathrm{f} \% \mathrm{C} 3 \% \mathrm{BCr}$-November 987642983 / 988260302/?ov=aHR0cDovL3d3dy5sb3ZlbHlib29rcy5kZS9hdXRvci9JbW9nZW44Um9zZ S9EaWUtUG9ydGFsLUNocm9uaWdbi1Qb3J0YWwtQmFuZCOxLTk3NzY2MDY0My 13L2FrdHVlbGwv\&lisre=modern. 
die Geschichte klang interessant und doch da war sie, die Angst vor zu vielen Klischees. Denn diese Thematik leitet schnell dazu in Klischees zu verfallen und nicht neu zu denken und nur an der Oberfläche zu kratzen, sowie in Kitsch zu verfallen. Der Autorin ,Shilpi Somaya Gowda ist es nicht immer gelungen genau diesen Dingen aus dem Weg zu gehen, gegen Ende nahm in meinen Augen der Kitsch und die Rührseligkeit immer mehr Überhand und doch sind es genau die Stellen, an denen sie es schafft von diesen Klischees auszubrechen umso schöner. "28

Und die Buchkritik kommt nach weiteren Ausführungen zu dem bezeichnenden Schluss: „Das Buch lädt dazu ein, sich mit einer Tasse Tee einen gemütlichen Abend zu machen und in dieses Buch voller Differenzen einzutauchen." Genau darum geht es in den sozialen Netzwerken: Jemand hat mit Verve gelesen, taucht in die Geschichte ganz ein und reflektiert dabei zugleich das Leseerlebnis in einfachen und nachvollziehbaren Bewertungsmustern. Geschrieben ist das für Gleichgesinnte. Eben das ist das typisch moderne Leserverhalten seit Rousseau, eher identifikatorisch, aber auch kritisch, und adressiert an die jeweilige gleichgesinnte Gruppe. „Social Reading" nennen das die Verlage, was bei Rousseau Herzensergießungen hieß. Und auch bei Amazon gilt, dass nur der positiv von anderen Lesern gewertet wird, der mehr als bloße Geschmacksurteile abgibt. Man will eben nicht unbedingt neue Leser für ein Buch oder einen Autor gewinnen, sondern sich mit Gleichgesinnten einstimmen. Insofern sind diese Laienrezensionen vielleicht schlicht, aber sie sind nur die digitalen Herzensergießungen der Leser unserer Tage. Und sie sind eben viel zahlreicher als zu Rousseaus Zeiten.

Vielleicht gesteht man noch zu, solche Leserinnen der sozialen Netzwerke nicht zu verlachen, aber spätestens bei den Computerspielen ist der Feind des Buches doch nicht mehr zu leugnen, wird man einwenden. Doch auch hier lohnt es sich, wenigstens ein paar Unterscheidungen nicht aus dem Blick zu verlieren, bevor von den Abgründen die Rede sein muss. Zunächst einmal deutet einiges darauf hin, dass die Leidenschaft für Computerspiele mit dem Bildungsstand nicht fällt, eher umgekehrt. ${ }^{29}$ So kontraintuitiv wie die Zunahme der Intelligenz ist dieser Zusammenhang, dass gerade Jungens aus bücherreichen Haushalten intensive Computerspieler sind und das zumeist auch in Gruppen tun, weil die intelligenten Spiele eine gehörige Portion sozialer Intelligenz voraussetzen. Die Monster kön-

28 hıср://www.lovelybooks.de/auror/Shilpi-Somaya-Gowda/Geheime-Tochter-951212442-w/ rezension-987804841/.

29 Zu diesem Ergebnis kommt die Forsa-Umfrage im Auftrag des Branchenverbandes BITKOM, http://www.birkom.org/files/documents/BITKOM_Presseinfo_Nurzer_von_Computerspielen _29_07_2009.pdf. 
nen meist nur in einer sich gut abstimmenden Gruppe besiegt werden, wie etwa in World of Warcraft, dem derzeit größten Massen-MehrspielerComputerspiel, dessen Handlung in einer phantastisch-dystopen Welt spielt und mehr als 10 Millionen Spieler weltweit anzieht. In Deutschland sind die Spieler, soweit hier überhaupt belastbare Zahlen vorliegen, eher akademisch gebildet oder besuchen eher höhere Schulen. In den letzten Jahren nimmt der Anteil der Frauen unter den Spielern auffällig zu, vermutlich deshalb, weil in diesen Spielen eine Selbstwirksamkeit erfahren wird, die eben der Grund ist, warum wir lesen oder spielen, nämlich in einer Intensität handeln zu können wie sonst nur selten im Leben. ${ }^{30}$ Und vieles deutet darauf hin, dass gute Gamer über ein gesteigertes Reaktionsvermögen verfügen, besser im Multitasking, im parallelen Verarbeiten, in der räumlichen Orientierung und in sozialen Abstimmungsverhalten sind. Unter den fast 80 Prozent der 10-jährigen, die heute Zugang zum Internet haben und von denen mehr als 50\% auch Facebook-Mitglieder sind, haben diejenigen, die Compurer spielen noch am ehesten überhaupt eine Vorstellung davon, wie ein Computer funktioniert. Spielen macht nicht nowwendig dumm. Und wie die Jungen, so die Alten. Eine jüngste australische Srudie mit mehr als 5.000 Teilnehmern zwischen 69 und 87 Jahren, die deren Computernutzungsverhalten über mehrere Jahre mit NichtComputernutzern dieser Altersgruppe verglichen hat, legt nahe, dass die tägliche Nutzung von Email, Interner, Textrerarbeitung, Spielen und Netzwerken Demenzerkrankungen abzuschwächen scheint. ${ }^{3}$

Solche Ergebnisse sind mit einiger Vorsicht zu bewerten. Das gilt gleichermaßen, wenn von den Verlierern der digitalen Welt zu sprechen ist, jenen ca. 15 bis 20 Prozent, denen nicht vorgelesen wird, dic nicht lernen mit Medien umzugehen, sondern vernachlässigt vom vierten Stock aus auf laute Straßen schauen und dann in Computerspielen oder abgründigen Diskussionszirkeln mehr Ansprache finden als sonst irgendwo. Wer nicht gelernt hat, sich auf das Fahrrad zu schwingen, um einen Freund zu besuchen, nicht im Fußballverein, in der Theater-AG oder in einer $\mathrm{Mu}$ sikgruppe mitspielt und also nicht viel mehr kann als abzuhängen, findet in Computerspielen und im Internet jene Verstärkung, die asoziale Verhaltenseigenschaften fördert. Computerspiele können also sehr wohl für diese meist männlichen Jugendlichen, denen keiner vorliest, fatale Auswir-

\footnotetext{
30. Ryan Rigney, Why Shooting Games Make Your Brain Happy, in: Wired (26.7.2012), htcp:// www.wired.com/gamelife/2012/07/shoorers/.

31 Osvaldo P. Almeida et al, Older men who use compurer have lower risk of dementia, in: PLOS ONE 7, 8 (2012), e44239. doi:10.1371/journal pone,0044239, http://www.plosone. org/article/info\%3Adoi\%2F10.1371\%2Fjournal,pone.0044239.
} 
kungen haben. Sie treiben eine Brutalität unter die Oberfläche, die dann nur einen kleinen Anlass braucht, um zuzuschlagen. Sich ständig extremen Gewalt- und Sexdarstellungen auszusetzen hat hier fatale Folgen. Das gilt auch für die Nutzung sozialer Netzwerke. Facebook scheint ganz ähnlich katalytisch verstärkend zu wirken. Für diejenigen, die nach Anerkennung suchen, die sie sonst nicht bekommen, impulsiv sind, weil sie niemanden haben, der egal, was sie machen, zu ihnen hält, für die sind Computerspiele und ist auch Facebook eine dauernde Ablenkung nach unten, die eine Abwärtsspirale nur beschleunigt. Pseudo-Freundschaften werden in Zahlen von über 200 bis 400 ,Freunden' demonstriert, denen keine soziale Wirklichkeit entspricht. Das Abfilmen von Schlägereien und das dann in Netzwerken wie Facebook zu verbreiten, dient nur der Demütigung anderer. Suchtartiges Verhalten kommt da auf. ${ }^{32}$

Für die sozial Aktiven dagegen intensiviert Facebook auch deren reale Kontakte. ${ }^{33}$ Insofern reflektieren Facebook-Profile die aktuellen Persönlichkeitsmerkmale, die guten wie die schlechten. ${ }^{34}$ Wir wissen aus einer Reihe von Studien, dass Medien nicht neue Persönlichkeitsmerkmale erfinden. Aber vorhandene Persönlichkeitsmerkmale können sehr wohl verlagert oder verstärkt werden. Darin sind Medien gefährlich ${ }^{35}$ oder auch nützlich. Lässt man Leute Filme sehen oder Bücher wie Harry Potter lesen, in denen Magie vorkommt, und testet sie danach, für wie plausibel sie es halten, dass vielleicht doch ein bisschen Magie auch im wirklichen Leben möglich wäre, dann steigt die Vermutung, das könnte doch ein bisschen möglich sein im Vergleich zu Gruppen, die nicht Harry Potter gesehen oder gelesen haben. Lässt man Leute dagegen Geschichten von Hooligans lesen, die nur auf die nächste Schlägerei warten und keine schwierigere Aufgabe zu lösen haben als das Öffnen einer Bierflasche, dann schneiden diese Leser im Vergleich mit Kontrollgruppen in Intelligenztest signifkant schlechter ab. Man kann sich auch dumm lesen, ob mit Büchern, Computerspielen

32 Vgl. z. B. das Daren- und Faktenblatr der Drogenbeauftragren der Bundesregierung vom 9.10.2012, http://www.drogenbeauftragte.de/fileadmin/dateien-dba/Presse/Downloads/Hand out_PK_Jahrestagung_2012.pdf oder den Bericht von Hans-Jürgen Rumpf et al., Prävalenz der Internetabhängigkeit, 2011, http://www.drogenbeauftragte.de/fileadmin/dateien-dba/ DrogenundSucht/Computerspiele_Internersucht/Downloads/PINTA-Bericht-

Endfassung_280611.pdf.

33 Adriana M. Manago, Tamara Taylor, Parricia M. Greenfield, Me and my 400 friends. The anatomy of college students' Facebook networks, their communication parterns, and well-being, in: Developmental Psychology 48, 2 (2012), S. 369-380.

34 Mitja D. Back et al., Facebook Profiles Reflect Actual Personality, Nor Self-Idealizarion, in: Psychological Science 21 (2010), S. 372-374.

35 Craig Anderson, Douglas Gencile, \& Katherine Buckley, Violent video game effects on children and adolescents. Theory, resedrch, and public policy, Oxford 2007. 
oder Facebook. Aber das sind temporäre Verlagerungen von Persönlichkeitsmerkmalen und sie wirken dauerhaft nur dann, wenn sie mit anderen sozialen Entwertungen regelmäßig zusammengehen. Computerspiele sind also gefährlich für diejenigen, die schon gefährdet sind, die keiner in den Arm nimmt. Es wundert dann nicht, dass unter den Jugendlichen in Deutschland mehr als 40 Prozent in Büchern und Tageszeitungen blättern und erwa die Hälfte der Jugendlichen eine Tageszeitung noch immer für das glaubwürdigste Medium hält, noch vor Fernsehen, Radio und Internet. ${ }^{36}$ Die einen gewinnen durch die neuen Medien, die anderen verlieren deutlich. ${ }^{37}$ Die schiere Exposition drastischer Gewalt- und Sexdarstellung wirkt auf diese Gruppe verheerend, ${ }^{38}$ während sonst die Gewalt in Gesellschaften wie der deutschen eher abnimmt. Und das, obgleich nicht nur in Computerspielen, sondern selbst in der Hochliteratur die Darstellungen von Gewalt und Sexualität deutlich zugenommen hat. Wie der Sozialpsychologe Christopher J. Ferguson anmerkt, berücksichtigen die Studien, die direkt vom Compurerspielen auf einen Anstieg gewalttätigen Verhaltens schließen, jene Faktoren von Vernachlässigung und Gewalt in Familien nicht, die zu berücksichtigen wären." Sonst könnte man auch nicht erkiären, warum einerseirs fast jeder Junge heute Computer spielt, aber die Gewalt deshalb niche nach oben geschnellt ist.

Anders gesagt: Compurer erserzen keine Eltern, keine Freunde und keine Lehrer. Im Gegenteil, denn die da so .rwittern', gamen' oder ,liken', haben von sich aus wenig Ahnung davon, wie mit Computer und Internet umzugehen ist. Wenn ihnen das niemand beibringt, wenn niemand sagt, dass jetzt genug Zeit vor dem Rechner verbracht worden ist, dann kann man auch vor dem Computer dumm werden, das ist keine Frage. Aber das ist nicht auf den Computer beschränkt, noch hängt es am Übergewicht des Visuellen, das man dem Computer nachsagt. Auch Bilderbücher ersetzen ja nicht das konzeptuelle Begreifen der Welt. Und doch würde niemand Bilderbücher verurteilen, sondern sagen, dass visuelle, körperliche und intellektuelle Erfahrung mit der Welt gleichermaßen eingeübt werden müssen. Das gilt immer noch, zumal in einer Welt, die eher komplexer als

\footnotetext{
36 Thomas Rathgeb für den Medienpädagogischen Forschungsverbund Süidwest, $/ 1 M$ 2012. Jugend, Information, (Multi-)Media. Basisstudie zum Medienumgang 12-bis 19-Jähriger in Deutschland, 2012, hıр://www.mpfs,de/fleadmin/JIM-pdf12/JIM2012_Endversion.pdf

37 Esther Köhler, Computerspiele und Gewalt. Eine psychologische Entwarnung, Heidelberg 2008.

38 Ross O'Hara er al., Greater exposure to sexual content in popular movies predicrs earlier sexual debur and increased sexual risk taking, in: Psychological Science 23 (2012), S. 984-993.

39 Christopher J. Ferguson, Video games don't make kids violent, in: Time (7.12.2011), hrtp:// ideas.time.com/2011/12/07/video-games-dont-make-kids-violent/.
} 
einfacher wird, in der viel schneller visuelle und intellektuelle Anforderungen ineinander übergehen. Das nennen wir Erziehung und die fängt immer noch mit dem abendlichen Vorlesen für unsere Kinder an.

\section{Medien und kein Ende}

Damit komme ich zum Schluss, zum Ausblick auf die Zukunft des Buchs und seiner Leser im digitalen Zeitalter. Da die Zukunft notorisch ungewiss ist, kann es nur um Vermutungen gehen, die dann meist übersehen, dass jemand auf die Idee zum Beispiel des Internets kommen kann und dann alles doch ganz anders kommt. Das bisher Vorgerragene läufr immer wieder darauf hinaus, dass in der Moderne mehr Bücher, ob gedruck oder digiral, mehr Genres, mehr Medien und vor allem mehr Leser zusammentommen. Es spricht einiges dafür, dass wir nicht mehr in einer Kultur leben, die als Buchkultur angemessen beschrieben wäre. Eine Lesekultur dagegen haben wir mehr denn je. Das fängt beim Vorlesen an, denn hier sind schon ganz unterschiedliche Bücher in den Händen der Eltern und Kinder, gedruckte und E-Books, Tablets und Kinderbuch-Apps. ${ }^{40}$ Ein erster Befund lautet einmal mehr, dass der Gegensatz zwischen gedrucktem und digitalem Lesen für die großen und kleinen Leser gar nicht wichtig ist. Nur die Medienkritik hält an ihm beharrlich fest. Wenn aber nicht mehr das Buch unsere Kultur integriert, sondern sehr unterschiedliche Bücher, die oft gar nicht mehr wie Bücher aussehen, dann steht zu erwarten, dass zum Lesen im digitalen Zeitalter gehört, in verschiedenen Formaten lesen zu können. Diese Fähigkeit wird in den nächsten Jahren vermurlich noch mehr gebraucht werden als jetzt schon. Und dass dies kein einfacheres Lesen ist, steht zu erwarten.

Zunehmen wird wohl auch das identifikatorische Lesen, nur wird es nicht auf das gedruckte Buch begrenzt bleiben. Gerade die Art zu lesen, die sich erst in der Neuzeit herausgebildet hat, sucht sich schneller neue Formate, Gattungen und Medien - und findet sie. Denn das digitale Zeitalter erlaubt es, nicht nur zwischen den Buchtiteln leichter zu wechseln, sondern auch für den Hunger nach Geschichten, den einmal Karl Mays Romane erfüllt haben, heute in ganz unterschiedlichen Medien fündig zu werden. Das Buch wird ein noch engerer Freund auch in dem Sinne, dass es uns regelrecht kennt. Als digitales Buch kennt es etwa unsere Vorlieben auch etwa für bestimmte Drucktypen. Diese Kindle-Leserin mag vielleicht eine serifenreichere Schritt, der technikaffine Leser eher Arial. Auch weiß unser Buch, dass unsere Augen an Sehkraft nachlassen und stellt schon die

40) Vorlesestudie 2012 der Stiftung Lesen, http://www.stiftunglesen,de/vorlesesrudie-2012. 
Schriftgröße so ein, dass wir nicht nach der Brille suchen müssen. Wo früher nur ein schmaler Rand für eigene Notizen blieb, kann ich in digitale Bücher seitenlange Bemerkungen einschieben, Zeichnungen ablegen und verwandte Dokumente mit meinem ganz individualisierten Buch verknüpfen. Mehr noch ich kann auch mit meinem Buch reden und ihm zuflüstern, wie sehr ich um Anna Karenina weine, und mein Buch zeichnet dann diese meine Empfindungen auf. Vielleicht wird schon in nächster Zukunft mein Buch dann aus der erfundenen Welt antworten, wenn ich mit ihm spreche. So ist mein Buch bald schon mein Freund, wie es das 18. Jahrhundert ausgedrückt hätte. ,Living books' nennt es unsere Zeit und das nutzen die jungen, aber immer mehr auch die alten Leser. Die digitale Modernisierung geht weiterhin in den Spuren der modernen Leserevolution, nur die Gangart könnte schneller werden.

Schon unsere Kinder wechseln mit einer nie zuvor gekannten Behändigkeit zwischen den Büchern, den digitalen Medien und den Sprachen dieser Welt. Sie verknüpfen Buch, Film und Lego-Spielzeug und verkleiden sich als Harry Potrer und Hermine Granger, als wäre es die größte Selbstverständlichkeit der Welt. Die da lesen. spielen auch und schauen auch, aber sie schreiben auch, drehen Filme und programmieren mir Programmen wie ,Scrarch Compurerspiele selbst. Prosumer nennt man diese Verbindung von Konsument und Produzenten in der digitalen Welt. Sie erlaubt es, mit einer bisher nicht gekannten Geschwindigkeir, neue Genres und neue Gattungen aus der Kreuzung bekannter herzustellen. Parodierende YoutubeFilme auf alle möglichen Medienereignisse sind heute schon millionenfach zu finden. Die Digitalisierung des Lesens multipliziert dieses Surfen zwischen den Medien und Formaten, einfach weil alles am Ende digitale Daten sind, die fast ohne Grenze ineinander überführbar sind. Cervantes, der sich kaum vorstellen konnte, dass sein Roman ohne sein Zutun weiter gedichtet werden würde, hätte sich nicht träumen lassen, wie behänd heute schon Kinder weiterschreiben.

Zugleich sind schon heute in Google Books oder in der Europeana ganze Kontinence bislang schwer zugänglicher Bücher und kultureller Dokumente in einer Weise zugänglich, die noch vor Kurzem kaum vorstellbar war. Auch das mag unsere digitale Lesekultur dann von der gedruckten unterscheiden, diese unglaubliche Zahl der Bücher, auch und gerade der einmal gedruckten, die nur einen Mausklick entfernt sind. ${ }^{41}$ Vermutet werden kann daher, dass die unsere vertraute (Buch-) Kultur sich auch dahin ver-

41 Adriaan van der Weel, Changing our textunl minds. Towards a digital order of knowledge, Manchester 2011. 
ändern wird, dass sie ganze Ländereien alter und ferner Bücher verknüpft. Und wenn die automatisierte Übersetzung bald soweit ist, dass ein japanischer Roman des Mittelalters in wenigen Sekunden ins Finnische oder Deutsche übersetzt ist, dann kommt es zu ganz anderen Aushandlungen über das, was unsere Kultur ausmachen soll. Die Landkarte unserer Kultur wird also schneller als bislang bekannt in Bewegung kommen, die Kontinente werden sich schneller als zuvor verschieben und zugleich wird der Bedarf wachsen, festes Ufer zu haben. Lesen im digitalen Zeitalter ist also in einer nie gekannten Breite und Tiefe möglich. Auch das verläuft in den Bahnen der Moderne, nur schneller und diversifizierter, und entspricht auch der wachsenden Komplexität unserer Arbeitswelt, die immer weniger einfache Arbeiten noch übrig lässt. Der immer noch viel zu hohe Anteil funktionaler Analphabeten passt dazu immer weniger.

Und noch einmal: Vieles ist schlimmer Unfug, Verantwortungslosigkeit und grob fahrlässig: Fernsehen für Kleinkinder etwa, tägliches 7-bis 8-stündiges Abkapseln vor der Welt, sei es mit Büchern, Fernsehern und Computer, oder Jugendliche permanent exzessiven Gewaltszenen auszusetzen. Erziehung wird nicht überflüssig werden, gerade in einer fundamentalliberalisierten Gesellschaft.

Ob aus dieser Dynamik, ja vielleicht sogar Explosion der Lesewelten ein qualitativ anderes Lesen einmal wird, wissen wir nicht. Abzusehen ist freilich schon heute, wie sehr sich die Grenzen zu anderen Formaten verlieren, sei es zu den visuellen, sei es auch bald schon zu den virtuellen. Der Hunger nach Geschichten von Rosamunde Pilcher bis Franz Kafka wird nicht weniger werden, die digitalen Herzensergießungen werden immer bunter fließen, Formate, die wir uns heute nicht vorstellen können, werden dazukommen, so dass wir uns schon heute anstrengen müssen zu überlegen, wie die Welt eigentlich vor zehn Jahren aussah. Vielleicht geht also geht gerade das Zeitalter des Buchs zu Ende, das des Lesens geht aber weiter. Denn was ziemlich gleich bleibt, das sind wir, die Leser. 